\title{
Modelación del contenido de agua de los suelos y su relación con los incendios forestales en la Sierra Madre Occidental de Durango, México
}

\section{Modeling of the water content of soils and its relation with wild fires in the Mountain Sierra Madre range of Durango, Mexico}

\author{
José de Jesús Návar Cháidez1
}

\begin{abstract}
RESUMEN
Las técnicas que predicen el riesgo de incendios forestales utilizan un submodelo hidroclimático. En esta investigación se probaron los efectos de la precipitación, de la evaporación medida en tanques (Et), de la evapotranspiración potencial estimada, Etp) y del contenido de humedad del suelo $(\theta)$, con el número de incendios y la superficie forestal incendiada del estado de Durango, México. Para el cálculo de Etp y de $\theta$ se aplicó un modelo físicamente basado en la estimación del balance del agua de los suelos forestales que se alimenta con variables climáticas, de cobertura forestal, edafológicas y de ponderación. Además, se relacionó el fenómeno climático El Niño con las variables de los incendios forestales previamente señalados. La humedad del suelo estimada con el modelo hidrológico resultó ser mejor predictora del número de incendios y de la superficie forestal incendiada que los factores climáticos simples. El índice del fenómeno climático El Niño, expresado durante noviembre-diciembre del año inmediatamente anterior, explica por arriba del $30 \%$ en la superficie forestal incendiada durante la etapa seca de enero-mayo de los bosques templados. Se recomienda emplear el modelo físico de suelos forestales como parámetro hidrológico porque se encuentra estrechamente relacionado con la humedad de los combustibles en la estimación del riesgo de incendios forestales en los bosques templados.
\end{abstract}

PALABRAS CLAVE:

Balance hídrico de suelos, distribución binomial negativa, modelos físicamente basado, número de incendios, superficie forestal incendiada, regresión.

\begin{abstract}
Mathematical techniques that predict the risk of forest fires use a hydro-climatic sub-model, which is generally fed with simple climatic variables such as rainfall, potential evapotranspiration, or the air relative humidity. In this research we report the effect of rainfall, pan evaporation (Et), and potential evapotranspiration (Etp), and the soil moisture content $(\theta)$, were related with the number of annual forest fires and the area annually burned by forest fires in the state of Durango, Mexico. A hydrologic, mass balance, physically-based model that feeds on climate, soil and plant cover variables as well as weighting factors was employed to calculate Etp and $\theta$. In addition, the El Niño index was statistically related to the forest fire variables described above. The $\theta$ variable resulted to be better related than the simple climatic variables to the number of forest fires and the area burned by forest fires. El Niño index expressed during the November-December phase of the year $\mathrm{t}-1$ explains above
\end{abstract}


$30 \%$ of the total burned area variation during the dry season of January-May of temperate forests. It is recommended to use the soil moisture content estimated by the water balance as predictor variable in the fire risk assessment of temperate forests.

KEY WORDS:

Water balance of soils, negative binomial distribution, physically based model, numbers and area burned by forest fires, regression.

\section{INTRODUCCIÓN}

El cálculo del área incendiada futura, la intensidad y comportamiento del fuego y otras características de los incendios forestales son fenómenos complejos. Los principios básicos indican que para que exista un incendio forestal se necesita de: a) combustibles con bajo contenido de humedad, b) oxígeno provisto por el aire, c) la fuente de ignición (Johnson y Miyanishi, 2001).

Estos componentes han sido compilados en las nuevas tecnologías de predicción del número y superficie forestal incendiada, de tal manera que actualmente existen diversos modelos en la literatura universal para predecir el riesgo de incendios forestales, dentro de los cuales destacan: a) el Canadian Forest Fire Danger Rating System (http://fire.cfs.nrcan.gc.ca); b) el Sistema Integrado de Italia (Fiorucci et al., 2004); c) el Instituto Meteorológico Portugués (Bugalho y Pessanha, 2007), d) el Sistema CFS-Conafor para México (Conafor, 2008), entre otros. Todos los sistemas incluyen generalmente dos componentes, el índice meteorológico y el sistema de previsión del comportamiento del fuego.

El índice meteorológico incluye generalmente los parámetros de humedad de los combustibles ligeros, del humus y de sequía, entre otros parámetros que gene- ralmente se integran en uno solo. Estos valores de manera normal se extrapolan de datos climatológicos provenientes de registros instrumentales, dentro de los cuales se incluyen: mediciones diarias de temperatura, la humedad relativa del aire, la velocidad y dirección del viento y la precipitación acumulada durante 24 horas (http://fire.cfs.nrcan.gc.ca). Sin embargo, pocos estudios consideran los balances hidrológicos con componentes o submodelos físicamente basados como variables de entrada en el subsistema climático para predecir el riesgo de incendios forestales en un bosque determinado (Lawson et al., 1997). Por esta razón, la investigación tuvo los siguientes objetivos.

\section{OBJETIVOS}

Probar si las variables Etp y $\theta$, provenientes de un balance hidrológico, proveen mejores estimadores del número de incendios y de la superficie forestal incendiada, que los factores climáticos convencionalmente empleados.

Probar el efecto climático de El Niño, en las variables de incendios señaladas anteriormente para el estado de Durango, México.

\section{MATERIALES Y MÉTODOS}

El estudio se realizó en el estado de Durango, México. Se localiza en la porción centro-norte de México (Fig. 1), dentro de las coordenadas extremas, en su posición septentrional, 22035' LN y $104^{\circ} 50^{\prime}$ LW; en su zona oriental $24^{\circ} 44^{\prime}$ LN y $22^{\circ} 58^{\prime}$ LW; en su posición más al norte $26^{\circ} 83^{\prime}$ LN y $104^{\circ} 27^{\prime}$ ' LW y en su posición occidental $23^{\circ} 52^{\prime}$ LN y $107^{\circ} 21^{\prime}$ LW. Colinda por el norte con los estados de Chihuahua y Coahuila; al este con Coahuila y Zacatecas, al sur con Zacatecas y Nayarit y al oeste con Sinaloa y 


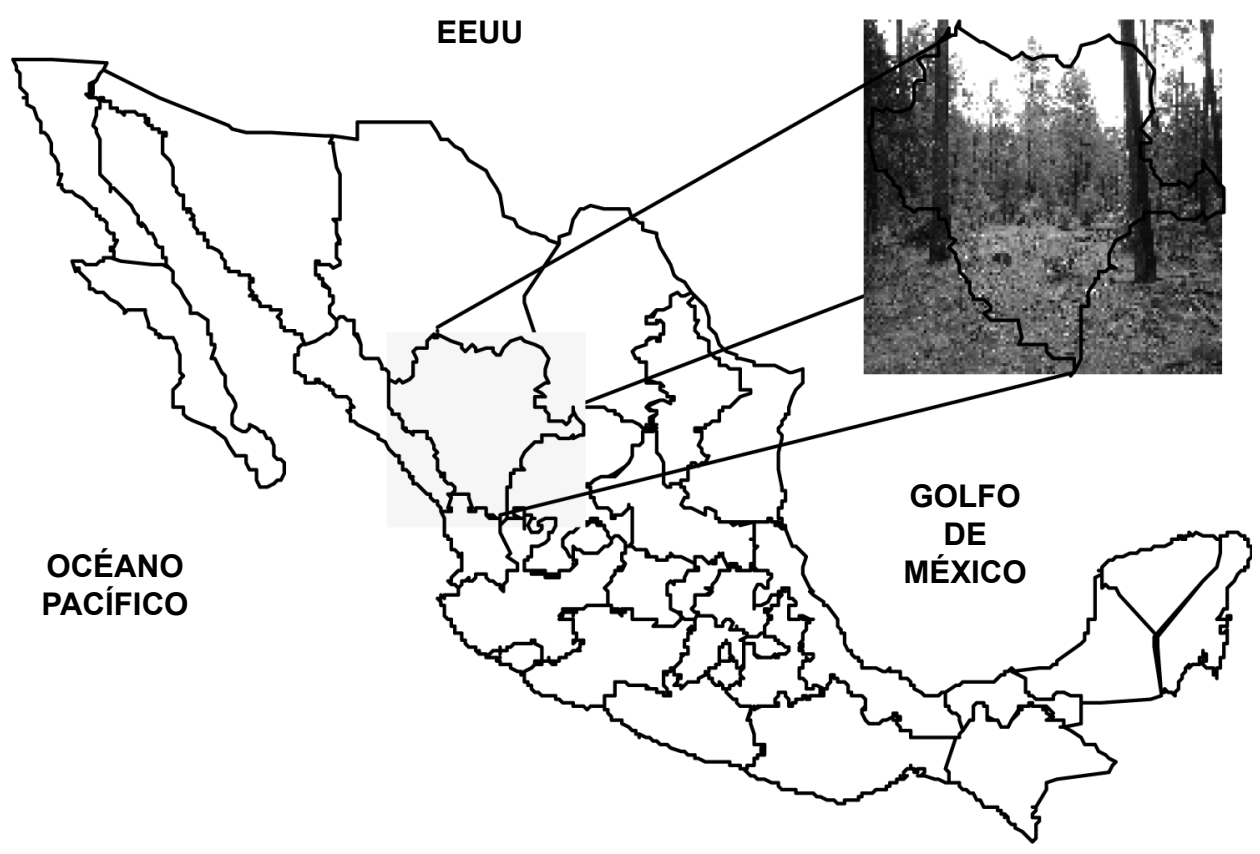

Figura 1. Ubicación del estado de Durango en México.

Nayarit. Se encuentra dentro de tres regiones fisiográficas: a) la vertiente del Océano Pacífico, b) la Sierra Madre Occidental y c) el desierto chihuahuense (INEGI, 2010).

Por su posición geográfica, su ubicación central en el país, la cercanía del Océano Pacífico y la presencia de la Sierra Madre Occidental y el Altiplano Mexicano, el estado de Durango posee una variedad de climas que van desde los áridos (BS) al centro-este del estado, los semiáridos (BS) en la región de los valles centrales y en la vertiente del Océano Pacífico, los semicálidos $((A) C)$ en la región del desierto chihuahuense y la vertiente del Océano Pacífico, hasta los templados (C) en la Sierra Madre Occidental, de acuerdo con la clasificación climática de Köppen (García, 1987).
Los tipos de vegetación dominantes son los bosques templados (4 700000 ha) seguido de los matorrales (2900 000 ha), los pastizales (1 000000 ha), la agricultura (1 300000 ha), las selvas bajas (500 000 ha) y la superficie restante (2 000000 ha) de otros tipos de vegetación. Los suelos predominantes son los litosoles $(37 \%)$, los regosoles $(30 \%)$ y los xerosoles (12\%). Esto se debe fundamentalmente a la topografía accidentada del territorio, a la geología y a la aridez del clima.

\section{El balance hídrico}

Se calcularon los cambios de agua para los suelos forestales de la Sierra Madre Occidental. Estos se pueden estimar por diversas metodologías, aquí se utilizó un ejemplo de un método físico indirecto, 
clasificado como Técnica del Balance de Masas (Viessman et al., 2007). La entrada del agua por la lluvia que se almacena en el suelo es el excedente de la precipitación que logra traspasar la barrera de la intercepción. El agua excedente se utiliza para humedecer el suelo y cuando éste se satura, el agua por arriba de la capacidad de campo puede escurrir súper o sub-superficialmente $y$ una parte puede percolarse hacia el acuífero. Las pérdidas del agua precipitada fueron clasificadas en este estudio por: a) la intercepción y b) la evapotranspiración, aunque en algunos casos la intercepción es tratada como parte de la evapotranspiración. La precipitación excedente, menos las pérdidas por escorrentía superficial y subterránea resulta en c) los cambios en el almacenamiento del agua del suelo. El suelo posee un almacén de agua dado por el sistema poroso; el exceso de agua o el agua gravitacional es aquella que se encuentra entre la capacidad de campo y el punto de saturación o con menos de $33,3 \mathrm{kPa}$ (menos de $1 / 3$ de bar) de presión; puede escurrir subsuperficialmente hacia el río o se percola hacia las capas profundas del suelo por la presencia de estructuras macroporosas para contribuir a la recarga de acuíferos. La ecuación del balance de masas dentro del suelo se presenta en los modelos [1], [2], [3], [4] y [5].

$$
\begin{gathered}
E-S=\frac{\partial A}{\partial t} ; E=P \\
S=(I+E v+T r+Q s+Q p) ; E t=(E v+T r) \\
P-(I+E t+Q s+Q p)=\frac{\partial A}{\partial t}
\end{gathered}
$$

$$
\begin{gathered}
Q=Q s+Q p \\
Q=P-(I+E t) \pm \frac{\partial A}{\partial t}
\end{gathered}
$$

La precipitación, $\mathrm{P}$, es la entrada del agua, E, al ecosistema y se registra normalmente en pluviómetros. La salida del agua fuera del suelo, S, se da por la interceptación, I, la evapotranspiración, Et, que es la suma de la evaporación del agua del suelo, Ev, y la transpiración, Tr, y la escorrentía superficial, Qs, y la percolación profunda que llega al acuífero, fuera de la zona de las raíces, Qp. La interceptación, I, es el agua que se utiliza en humedecer la vegetación y el mantillo y regresa a la atmósfera sin haber contribuido al aumento de la humedad del suelo. Esta es una pérdida de agua y en este caso se estima con el modelo modificado de Gash et al. (1995), cuya ecuación se presenta en el modelo [6]

$$
\begin{gathered}
\left.\sum_{j=1}^{n+m} I_{j}=n(c) P_{G}^{\prime}+\left(c \bar{E}_{c} / \bar{R}\right) \sum_{j=1}^{n}\left(P_{G j}^{\prime}-P_{G}^{\prime}\right)^{\cdot} 6\right] \\
+(c) \sum_{j=1}^{m} P_{G J}+q S_{c}+p_{t} \sum_{j=1}^{n-q} P_{G J}
\end{gathered}
$$

Donde:

$c=$ proporción del suelo sin cobertura forestal.

$\mathrm{Ec}=$ la tasa de evaporación del agua interceptada.

$\mathrm{R}=$ la intensidad de la lluvia sobre la cobertura.

$\mathrm{P}_{\mathrm{G}}{ }=$ cantidad de lluvia necesaria para iniciar la redistribución de la lluvia sobre el suelo.

$Q=$ la precipitación de lluvias que no sobrepasan $\mathrm{P}_{\mathrm{G}}$.

$\mathrm{S}_{\mathrm{C}}=$ la cantidad de agua necesaria para saturar la cobertura. 
$P_{t}=$ la proporción de la lluvia que es escorrentía cortical.

Los detalles de este modelo se pueden revisar en Návar y Bryan (1994) y Návar et al. (1999 y 1999a) y los parámetros para bosques mixtos, bosques de encino y bosques de pino se pueden consultar en Návar (2011; 2011a).

Un estimador de Et se calcula midiendo convencionalmente la evaporación en evaporímetros y la ponderación (Etp), se calcula con un factor de evaporación en tanques $(\mathrm{Et})$, un factor climático (Fc), un factor de cobertura forestal (Ftv) y finalmente con el almacén de agua del suelo dado por los contenidos iniciales de agua del suelo $(\theta \mathrm{i})$, el contenido de agua del suelo al punto de marchitamiento permanente $\left(\theta_{\mathrm{pmp}}\right)$ y el contenido del agua del suelo a la capacidad de campo $\left(\theta_{\mathrm{cc}}\right)$, a partir del modelo [7]:

$E t p=\frac{\operatorname{Ln}\left[100 \cdot \frac{\theta_{i}-\theta_{p m p}}{\theta_{c c}-\theta_{p m p}}\right]}{\operatorname{Ln}(101)} \cdot E t \cdot F c \cdot F t v$

El modelo [7] estima la evapotranspiración potencial, la cual está regulada por el contenido del agua del suelo y el tipo de cobertura forestal. Los detalles y factores de ponderación de la ecuación [7] se pueden encontrar en libros básicos de física de suelos (Hillel, 1980). Las formas de estimar estos parámetros son también parte de la física de suelos.

El contenido final del agua del suelo, en forma proporcional, se estima a partir del balance hídrico, donde $\theta_{\mathrm{i}}=\theta_{\mathrm{i}-1} \pm$ [Precipitación $n_{i-1}-$ Interceptación $_{i-1}-$ Etp]; i-1 se refiere al tiempo inmediato anterior, que para este propósito en la escala temporal diaria es del día anterior.
El modelo se alimentó con los siguientes parámetros del suelo: profundidad del suelo $=30 \mathrm{~cm}$, contenido del agua del suelo al punto de marchitamiento permanente $\left(\theta_{\mathrm{pmp}}\right)=0,15$; contenido del agua del suelo al punto de la capacidad de campo $\left(\theta_{\mathrm{cc}}\right)=0,45$, y contenido del agua del suelo al punto de saturación de la porosidad del suelo $\left(\theta_{\mathrm{s}}\right)=$ 0,50 ; con el parámetro climático de factor de conversión de Etp a Etr, Fc, =0,95; el parámetro de vegetación $(\mathrm{Ftv})=1,25$; y los parámetros de la interceptación (c) = 0,$058 ; E_{c}=2,64 ; R=12,66 ; P_{G}^{\prime}=0,25 ; q$ $=0,058 ; S_{c}=0,135 ; P_{t}=0,039$.

Los datos de la superficie y número de incendios forestales para el periodo de 1992 a 2006 se encontraron disponibles para relacionarlos con los parámetros resultantes, específicamente con el número de días que $\theta$ se encuentra por debajo de cierto límite, como un indicador de la sequía o del contenido de agua de los suelos y por lo tanto de la vegetación y del mantillo o combustibles que se encuentran en contacto con el suelo. Se utilizó la variable número de días con un $\theta$ por debajo de cierto umbral $x$ durante los meses de enero, febrero, marzo, abril y mayo. Este periodo es el de mayor presencia de incendios en Durango, dada una combinación de bajas precipitaciones acompañadas por fuertes vientos secos que reducen la humedad de los árboles, mantillo y suelos muy rápidamente.

El número y superficie forestal incendiada se relacionaron con el valor de $\theta$, con ecuaciones lineales y no lineales para determinar la ecuación de mejor ajuste. Se describen las ecuaciones y los estadísticos de ajuste coeficiente de determinación, error estándar y nivel de significancia probabilística.

Fulé y Covington (1997) reportaron datos sobre el número de incendios para los bosques templados del centro de 
Durango, México, para el periodo de 1945 a 1993. Esta información se utilizó también en la prueba de bondad de ajuste del valor estimado de $\theta$ por el balance hídrico. Los datos de Fulé y Covington (1997) presentaron $\mathrm{n}=$ número de incendios forestales $=15,12$ y 9 que quemaron: a) al menos un árbol, b) al menos $10 \%$ del arbolado y c) al menos $25 \%$ de los árboles presentes en un rodal. Se utilizó la distribución binomial negativa para estimar el número de años en los cuales existen incendios forestales para varios niveles de humedad.

\section{La distribución binomial negativa}

El número $X$ de ensayos realizados para producir k aciertos o éxitos, en un experimento binomial negativo se denomina variable aleatoria con una distribución binomial negativa. Si una repetición de eventos independientes resultan en un acierto o éxito, con una probabilidad $p o$ en un fallo o fracaso con una probabilidad $q=1-p$, entonces la distribución de probabilidad de la variable aleatoria $\mathrm{X}$, descrita como el número del intento en el cual ocurre el k-ésimo éxito o acierto es:

$$
n b(x ; r, p)=\left(\frac{x+r-1}{r-1}\right) p^{r} q^{x}
$$

La esperanza matemática y la varianza de la distribución binomial negativa $\mathrm{nb}(\mathrm{x}$; 1, p) están dadas por:

$$
E(x)=\mu=\frac{r(1-p)}{p}
$$

y

$$
V(x)=\sigma^{2}=\frac{r(1-p)}{p^{2}}
$$

La distribución binomial negativa estima los años en los cuales se presentan los incendios forestales con variantes en la variable $\theta$ proveniente del modelo hídrico. Los datos estimados por la distribución y los observados por Fulé y Covington (1997) sirvieron para determinar si $\theta$ explica parte de la variación aleatoria de los incendios forestales presentados en la fracción de la Sierra Madre Occidental de Durango, México.

Además, se ajustaron regresiones lineales y no lineales a los datos del número de incendios forestales y la superficie forestal incendiada con los factores climáticos simples (precipitación total anual, precipitación estacional acumulada (enero-mayo), evaporación, evapotranspiración y evapotranspiración ponderada estacional). Los datos de precipitación provienen generalmente de pluviómetros y los datos de evaporación provienen de evaporímetros de la estación climática $\mathrm{El}$ Salto, Durango, México. Los datos de Et y Etp provienen del modelo hídrico del balance de masas. Se utilizaron los datos climáticos de la estación de El Salto, Durango, México, porque se encuentra dentro de los bosques templados de la Sierra Madre Occidental de Durango, México. Los datos climáticos de la estación El Salto son representativos para todos los bosques templados de Durango, como se observará en los resultados. Esto sucede parcialmente porque las lluvias como las temperaturas que ocasionan la evapotranspiración ocurren principalmente por fenómenos climáticos de orden sinóptico, a escalas globales.

Además, se relacionó la superficie y el número de incendios con los eventos $\mathrm{El}$ Niño - Oscilación del Sur (ENSO por sus siglas en inglés: El Niño-Southern Oscilation) para el periodo de referencia señalado. Se ajustaron también ecuaciones de regresión para detectar cómo la presencia de este fenómeno climático ejerce una influencia en estos fenómenos. 


\section{RESULTADOS}

Los resultados anuales de la corrida del modelo con datos diarios se presentan en la tabla 1.

Los resultados muestran que la precipitación promedio anual para la región del Salto, Pueblo Nuevo, Durango, para el periodo de 1945 a 2003 es de 858 $\mathrm{mm}$, de los cuales, en promedio, 94 se interceptan, 603 se evapotranspiran y 146 $\mathrm{mm}$ producen caudales superficiales, subsuperficiales y subterráneos. Si se consideran los caudales totales como fuentes de salida de agua de los suelos, la descarga total que sale por los ríos se aproxima a $17 \%$ de la precipitación total. Este porcentaje es consistente con aqueIlos valores reportados por Návar (2008) en su balance hídrico para el estado de Durango, quien calculó que los caudales superficiales explican, en promedio, $15,5 \%$ de la precipitación promedio anual para el estado. De acuerdo con estima- ciones realizadas por el autor de esta investigación, entre $20 \%$ y $30 \%$ es caudal base y el restante es caudal directo. El contenido promedio del agua del suelo es de $0,28 \mathrm{~cm} \mathrm{~cm}^{-1}$ cuando el punto de saturación es de $0,50 \mathrm{~cm} \mathrm{~cm}^{-1} \mathrm{o}$ el contenido del agua a la capacidad de campo es de $0,45 \mathrm{~cm} \mathrm{~cm}^{-1}$. Del balance hídrico se extrae que los suelos permanecen la mayor parte del tiempo semisecos, con $\square$ cercanos al punto de marchitamiento; lo que explica la alta incidencia de incendios forestales y la falta de agua en el suelo como un factor limitante en la productividad forestal.

Los resultados de las relaciones entre el valor de $\theta$ proveniente del modelo del balance hidrológico expresado como sequedad del suelo para la época de incendios forestales y del número de incendios forestales para el estado para el periodo 1990-2002 se presentan en la figura 2 .

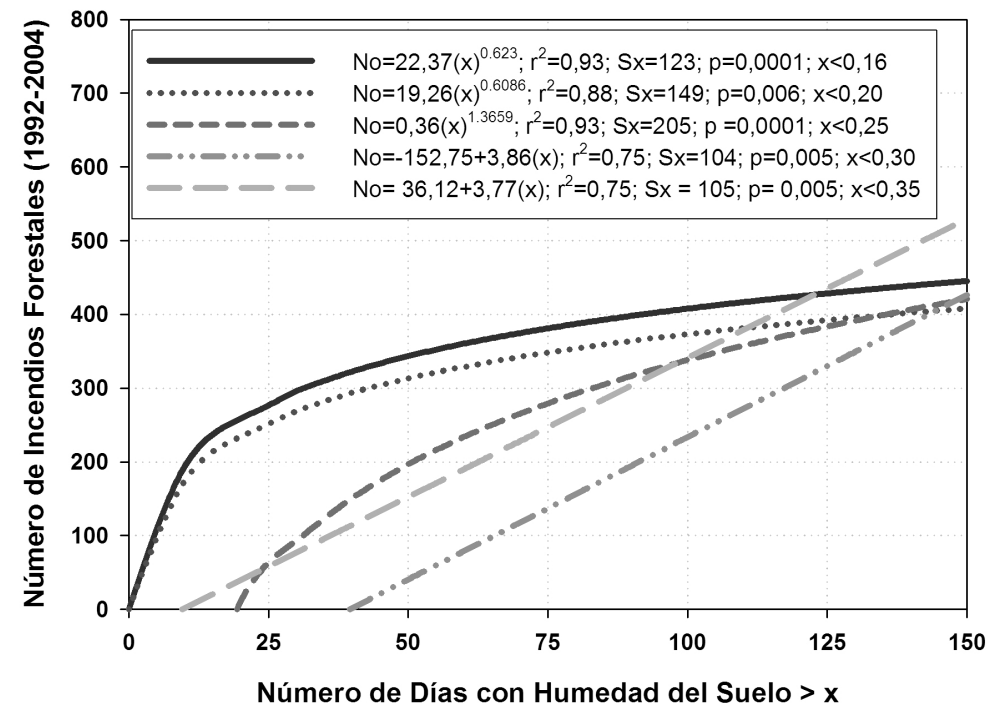

Figura 2. Relación entre el número de incendios forestales y el número de días por debajo de un valor de humedad del suelo $x$. 
Tabla 1. Variables del balance hídrico para suelos de la Sierra Madre Occidental del estado de Durango, México.

\begin{tabular}{|c|c|c|c|c|c|c|}
\hline Año & $\begin{array}{l}\text { Precipitación } \\
(P)(\mathrm{mm})\end{array}$ & $\begin{array}{l}\text { Interceptación } \\
\text { (I) }(\mathrm{mm})\end{array}$ & $\begin{array}{l}\text { Etp } \\
(\mathrm{mm})\end{array}$ & $\begin{array}{c}\text { Eta onderada } \\
\qquad(\mathrm{mm})\end{array}$ & $\begin{array}{c}\text { Theta } \\
\left(\mathrm{mm} \mathrm{mm}^{-1}\right)\end{array}$ & $\begin{array}{c}Q \\
(\mathrm{~mm})\end{array}$ \\
\hline 1945 & 827,50 & 90,80 & 1149,10 & 736,30 & 0,25 & 0,40 \\
\hline 1946 & 853,70 & 93,70 & 1135,50 & 722,00 & 0,25 & 38,10 \\
\hline 1947 & 902,40 & 99,00 & 1043,10 & 705,80 & 0,25 & 97,60 \\
\hline 1948 & 1012,90 & 111,00 & 1130,20 & 861,10 & 0,26 & 40,80 \\
\hline 1949 & 670,90 & 73,60 & 724,90 & 557,80 & 0,27 & 39,60 \\
\hline 1952 & 279,40 & 30,70 & 433,90 & 216,80 & 0,20 & 31,90 \\
\hline 1953 & 1022,00 & 111,90 & 1075,40 & 802,50 & 0,26 & 107,50 \\
\hline 1954 & 382,50 & 42,00 & 871,10 & 377,00 & 0,20 & 0,00 \\
\hline 1955 & 1007,50 & 110,30 & 1035,40 & 664,70 & 0,27 & 232,50 \\
\hline 1956 & 567,00 & 62,20 & 1013,90 & 467,50 & 0,21 & 37,30 \\
\hline 1957 & 555,00 & 60,80 & 767,80 & 461,50 & 0,23 & 32,60 \\
\hline 1958 & 158,20 & 17,30 & 284,90 & 144,10 & 0,21 & 0,00 \\
\hline 1959 & 457,10 & 50,10 & 814,30 & 386,30 & 0,20 & 20,70 \\
\hline 1960 & 759,00 & 83,10 & 915,10 & 598,90 & 0,26 & 77,00 \\
\hline 1961 & 741,70 & 81,20 & 883,50 & 608,40 & 0,26 & 52,20 \\
\hline 1962 & 880,00 & 96,40 & 925,70 & 650,90 & 0,26 & 132,70 \\
\hline 1963 & 1224,60 & 134,10 & 902,30 & 735,00 & 0,31 & 355,50 \\
\hline 1964 & 782,90 & 85,90 & 785,00 & 712,20 & 0,30 & 0,00 \\
\hline 1965 & 933,00 & 102,10 & 885,10 & 578,10 & 0,25 & 252,80 \\
\hline 1966 & 1235,10 & 135,20 & 833,50 & 805,80 & 0,33 & 294,10 \\
\hline 1967 & 1242,40 & 135,90 & 864,10 & 682,50 & 0,30 & 424,00 \\
\hline 1968 & 1413,40 & 154,50 & 875,40 & 811,60 & 0,33 & 447,30 \\
\hline 1969 & 871,80 & 95,40 & 834,50 & 681,00 & 0,29 & 95,40 \\
\hline 1970 & 883,00 & 96,60 & 873,20 & 678,30 & 0,29 & 108,10 \\
\hline 1971 & 705,50 & 77,20 & 929,70 & 604,10 & 0,24 & 24,20 \\
\hline 1972 & 1073,20 & 117,40 & 939,40 & 675,00 & 0,29 & 280,80 \\
\hline 1973 & 1044,50 & 114,30 & 906,00 & 756,90 & 0,29 & 173,40 \\
\hline 1974 & 1066,00 & 116,80 & 779,40 & 548,90 & 0,26 & 400,30 \\
\hline 1975 & 763,30 & 83,60 & 948,00 & 595,00 & 0,27 & 84,70 \\
\hline 1976 & 938,50 & 102,70 & 852,80 & 605,40 & 0,28 & 230,40 \\
\hline 1977 & 713,50 & 78,10 & 826,90 & 647,20 & 0,28 & 0,00 \\
\hline 1978 & 1162,80 & 127,20 & 884,30 & 627,20 & 0,26 & 408,40 \\
\hline 1979 & 643,30 & 70,40 & 674,60 & 453,80 & 0,24 & 119,20 \\
\hline 1980 & 769,90 & 84,20 & 638,50 & 479,70 & 0,29 & 206,00 \\
\hline 1981 & 1198,60 & 131,20 & 779,30 & 742,60 & 0,34 & 324,80 \\
\hline 1983 & 1102,80 & 120,90 & 839,50 & 806,40 & 0,34 & 175,50 \\
\hline 1984 & 755,20 & 82,90 & 851,30 & 527,20 & 0,25 & 145,10 \\
\hline 1985 & 1018,40 & 111,60 & 922,10 & 690,80 & 0,28 & 216,00 \\
\hline 1986 & 1098,00 & 120,30 & 902,10 & 688,70 & 0,28 & 289,00 \\
\hline 1987 & 406,90 & 44,60 & 255,00 & 262,90 & 0,36 & 99,40 \\
\hline 1988 & 868,50 & 95,20 & 999,60 & 713,40 & 0,27 & 59,90 \\
\hline 1989 & 785,00 & 86,10 & 935,70 & 626,80 & 0,26 & 72,10 \\
\hline
\end{tabular}


(finaliza Tabla 1)

\begin{tabular}{lcccccc}
\hline Año & $\begin{array}{c}\text { Precipitación } \\
(P)(\mathrm{mm})\end{array}$ & $\begin{array}{c}\text { Interceptación } \\
(\mathrm{I})(\mathrm{mm})\end{array}$ & $\begin{array}{c}\text { Etp } \\
(\mathrm{mm})\end{array}$ & $\begin{array}{c}\text { Eta onderada } \\
(\mathrm{mm})\end{array}$ & $\begin{array}{c}\text { Theta } \\
\left(\mathrm{mm} \mathrm{mm} \mathrm{mm}^{-1}\right)\end{array}$ & $\begin{array}{c}Q \\
(\mathrm{~mm})\end{array}$ \\
\hline 1990 & 136,00 & 14,90 & 242,00 & 171,70 & 0,26 & 0,00 \\
1991 & 1208,50 & 132,30 & 1012,50 & 639,10 & 0,27 & 437,10 \\
1992 & 1265,30 & 138,50 & 995,10 & 807,70 & 0,30 & 319,00 \\
1993 & 960,80 & 105,20 & 1002,30 & 711,30 & 0,26 & 144,30 \\
1994 & 961,70 & 105,40 & 1066,00 & 707,00 & 0,25 & 149,40 \\
1995 & 774,10 & 84,80 & 1055,00 & 568,80 & 0,23 & 120,50 \\
1996 & 910,80 & 99,80 & 960,90 & 617,90 & 0,25 & 193,20 \\
1997 & 999,50 & 109,60 & 923,70 & 858,80 & 0,30 & 31,10 \\
1998 & 660,60 & 72,40 & 926,40 & 597,40 & 0,25 & 0,00 \\
1999 & 770,00 & 84,06 & 409,92 & 409,92 & 0,38 & 103,15 \\
2000 & 786,50 & 85,83 & 459,03 & 459,03 & 0,40 & 94,36 \\
2001 & 938,00 & 102,41 & 450,34 & 450,34 & 0,40 & 130,75 \\
2002 & 943,00 & 102,99 & 525,27 & 525,27 & 0,41 & 112,74 \\
2003 & 953,00 & 104,07 & 524,88 & 524,88 & 0,41 & 112,74 \\
Promedio & 857,94 & 93,94 & 829,90 & 602,63 & 0,28 & 145,99 \\
Desviación & 270,11 & 29,54 & 221,15 & 164,04 & 0,05 & 128,22 \\
Intervalo & 70,75 & 7,74 & 57,92 & 42,96 & 0,01 & 33,58 \\
Coeficiente & & & & & & \\
De Variación & 31,48 & 31,45 & 26,65 & 27,22 & 18,24 & 87,83 \\
\hline
\end{tabular}

Donde: Etp=evapotranspiración potencial $(\mathrm{mm})$, Eta=evapotranspiración real $(\mathrm{mm})$; Theta=contenido promedio de humedad del suelo; $\mathrm{Q}=$ descarga de agua del suelo.

De la información de la figura 2 se extrae que el valor de $\theta$ explica entre $75 \%$ y $93 \%$ de la variación existente en el número de incendios forestales que se presentan en el estado. Esto demuestra la bondad del balance hídrico del suelo como estimador de este tipo de eventos. De las ecuaciones regresivas se extrae que el número de incendios crece exponencialmente en suelos demasiado secos $(\theta$ $<0,16 \mathrm{~cm} \mathrm{~cm}^{-1}$ ) y linealmente en suelos poco húmedos $\left(\theta>0,30 \mathrm{~cm} \mathrm{~cm}^{-1}\right)$. En suelos demasiado secos la ecuación alcanza una asíntota rápidamente, dada por los exponentes menores que 1,0 y se explica parcialmente por la falta de combustibles o de sistemas de ignición para continuar generando un mayor número de incendios forestales en estas condiciones de sequía. Otros factores que están dominando la asíntota pueden ser las variaciones del suelo que conllevan a mayores almacenamientos de agua que, eventualmente, necesitan de un mayor número de días con sequedad para generar un mayor número de incendios forestales. Entre estos factores se encuentran la profundidad por arriba de los $30 \mathrm{~cm}$, la cantidad de rocas dentro del suelo que produce cambios importantes en los balances hídricos de los mismos, la exposición que genera tasas diferenciales de evapotranspiración, la diversidad de especies de árboles que se encuentran en el rodal y que generan también tasas diferenciales de interceptación y de transpiración. 
La superficie forestal incendiada y su relación con el contenido de humedad del suelo se presentan en la figura 3. La variación en la superficie forestal incendiada explicada por los modelos de regresión varió desde $42 \%$ hasta $94 \%$. Estos modelos fueron significativos al 0,05 para suelos con humedades promedio por arriba de $0,25 \mathrm{~cm} \mathrm{~cm}^{-1}$ y al 0,10 para suelos por debajo de $0,25 \mathrm{~cm} \mathrm{~cm}^{-1}$. La superficie forestal incendiada aumenta linealmente con el número de días por debajo de la humedad $x$. Las líneas se desplazan hacia la derecha con el aumento en la humedad promedio del suelo, explicando que se requiere de mayor número de días para incendiar una misma superficie forestal anual. Por ejemplo, para alcanzar las 20000 hectáreas incendiadas, se requiere de 50,70 , 80 y 100 días con humedades del suelo promedio por debajo de $(0,16 ; 0,20 ; 0,25$ y 0,30$) \mathrm{cm} \mathrm{cm}^{-1}$, respectivamente. Los modelos casi convergen cuando se incendian las 40000 hectáreas, indicando que el contenido del agua del suelo en este rango de valores $(0,20 \leq \theta \leq 0,30) \mathrm{cm} \mathrm{cm}^{-1}$, es irrelevante en estas situaciones. Otros fenómenos operando individual o colectivamente explican en conjunto con humedades del suelo por debajo de $0,30 \mathrm{~cm}$ $\mathrm{cm}^{-1}$ la superficie forestal incendiada. Por ejemplo, durante 1998, cuando ocurrieron estos fenómenos que incendiaron por arriba de las $40 \quad 000$ hectáreas de bosques en el estado de Durango, se presentó una combinación de fuertes vientos secos provenientes del Océano Pacífico, combinado con una alta mortalidad de arbustos y árboles de algunas especies que sucumbieron a las fuertes heladas del 12 de diciembre de 1997.

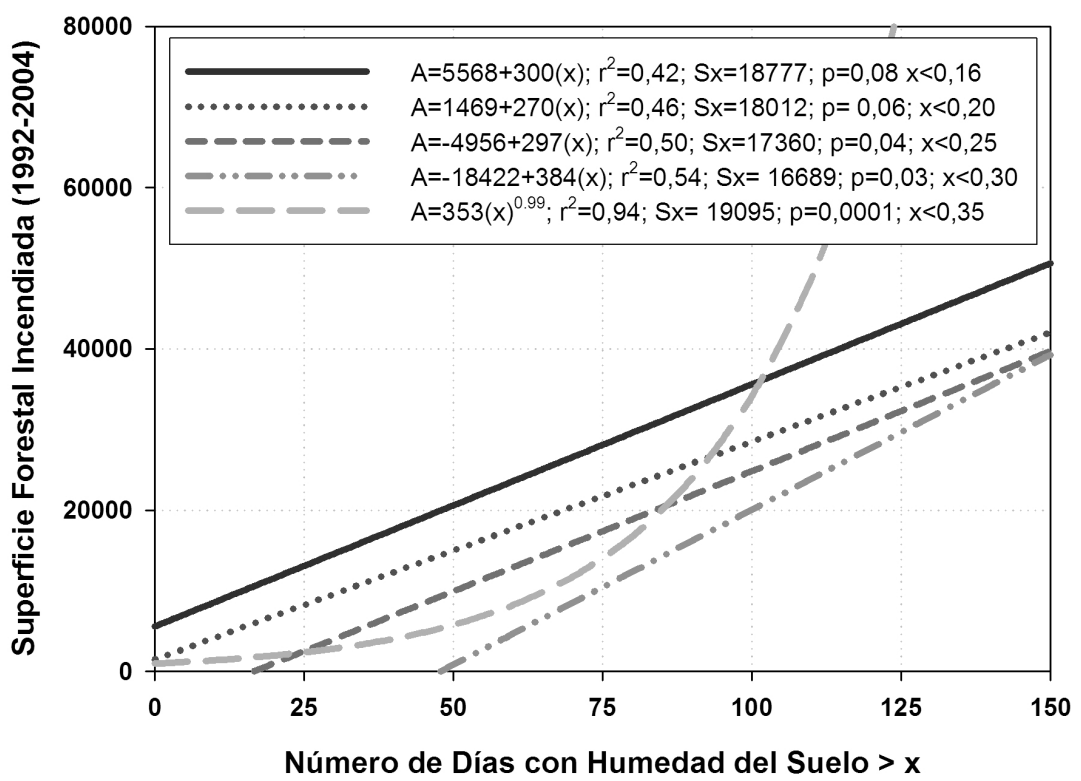

Figura 3. Relación entre la superficie forestal incendiada y el número de días por debajo de un nivel de humedad del suelo $x$. 
Los resultados del modelo hídrico conjuntado con los datos sobre incendios forestales observados por Fulé y Covington (1997), para los diferentes contenidos de humedad del suelo, se presentan en la tabla 2. La probabilidad de la presencia de incendios con $r=$ úmero de aciertos $\mathrm{y} x$ número de intentos necesarios para alcanzar el número de aciertos se presentan en la segunda parte de la tabla 2.

El modelo binomial negativo explica que el número de intentos para alcanzar el número de convergencia de incendios (observados y modelados) es claramente mayor (12-25) que el número de intentos observados por el modelo hídrico de predicción de la humedad del suelo (817). Por esta razón, probabilísticamente el modelo hidrológico explica por arriba de $66 \%(8 / 12$ y $17 / 25)$ de la variación en el número de incendios forestales en Durango, México. La consistencia del modelo se observa porque el número de días con humedad del suelo menores que 0,16 explican una mayor variación de la presencia de incendios $(8 / 15 ; 7 / 12 ;$ y $5 / 9)$ que a medida que el suelo se encuentra con un mayor contenido de humedad, $\mathrm{q}<0,20, \quad(4 / 15 ; 4 / 12 ;$ y $2 / 9)$ o $\mathrm{q}<0,25$, (5/15; 4/12; y $2 / 9)$.

El número de incendios y la superficie forestal incendiada no se encuentran estadísticamente relacionados con la precipitación anual o estacional, ni con la evapotranspiración anual, estacional o ponderada (Tabla 3). La escasa variación explicada por los modelos de regresión y la falta de significancia probabilística para las relaciones entre los factores del clima y el número de incendios y la superficie forestal incendiada, indican que se requiere de mayor información para poder calibrar estas asociaciones correctamente y poder justificar su presencia dentro de los modelos de riesgo de incendios forestales. Los valores anuales pueden no representar adecuadamente las condiciones climáticas presentes durante la época de incendios.

Las teleconexiones con el efecto climático El Niño, sobre la superficie forestal incendiada y el número de incendios es clara pero con una fuerte variación no explicada por las ecuaciones de regresión (Fig. 4). Los modelos lineales explican entre $15 \%$ y $38 \%$ de la variación total en el número de incendios y en la superficie forestal incendiada, respectivamente. Estadísticamente se predice de modo adecuado la superficie forestal incendiada por el fenómeno climático $\mathrm{El}$ Niño $(p=0,03)$ pero no el número de incendios forestales $(p=0,28)$. Si embargo, ambas ecuaciones poseen pendientes negativas; es decir, a medida que las aguas del Pacífico Sur Oriental se calientan por arriba de lo normal, el

Tabla 2. La distribución binomial negativa para estimar el número de aciertos, $r(8,8, y$ 5) con $p(r)=0,50$, con probabilidades $(0,995$ y 0,999$)$ de la presencia de incendios en un bosque templado de Durango, México.

\begin{tabular}{|c|c|c|c|c|c|c|}
\hline \multirow[t]{2}{*}{$\begin{array}{l}\text { Incendios } \\
\text { observados }\end{array}$} & \multicolumn{3}{|c|}{$\begin{array}{c}\text { Núm. de intentos, } \\
\text { con } P=0,0995 \text { que convergen } \\
\text { (del total) para humedades, } \theta\end{array}$} & \multicolumn{3}{|c|}{$\begin{array}{c}\text { Núm. de intentos, con } P=0,999 \\
\text { que convergen (del total) } \\
\text { para humedades, } \theta\end{array}$} \\
\hline & $\theta<0,16$ & $\theta<0,20$ & $\theta<0,25$ & $\theta<0,16$ & $\theta<0,20$ & $\theta<0,25$ \\
\hline 15 & $8(17)$ & $4(8)$ & $5(9)$ & $8(25$ & $4(17$ & $5(19)$ \\
\hline 12 & $7(17)$ & $4(8)$ & $4(9)$ & $7(23)$ & $4(17)$ & $4(17)$ \\
\hline 9 & $5(17)$ & $2(8)$ & 2(9) & $5(19)$ & $2(12)$ & $2(12)$ \\
\hline
\end{tabular}


Tabla 3. Coeficientes de determinación (la probabilidad de la significancia estadística) de la relación entre los parámetros climáticos individuales y el número de incendios y superficie forestal incendiada en el estado de Durango $(n=15)$.

\begin{tabular}{lcc}
\hline & Número de incendios & Superficie forestal \\
\hline Precipitación anual & $0,18(0,17)$ & $0,026(0,62)$ \\
Precipitación (enero-mayo) & $0,14(0,23)$ & $0,016(0,69)$ \\
Evapotranspiración anual & $0,014(0,71)$ & $0,0012(0,91)$ \\
Evapotranspiración (enero-mayo) & $0,13(0,26)$ & $0,016(0,69)$ \\
Evapotranspiración ponderada (enero-mayo) & $0,0074(0,79)$ & $0,017(0,69)$ \\
\hline
\end{tabular}

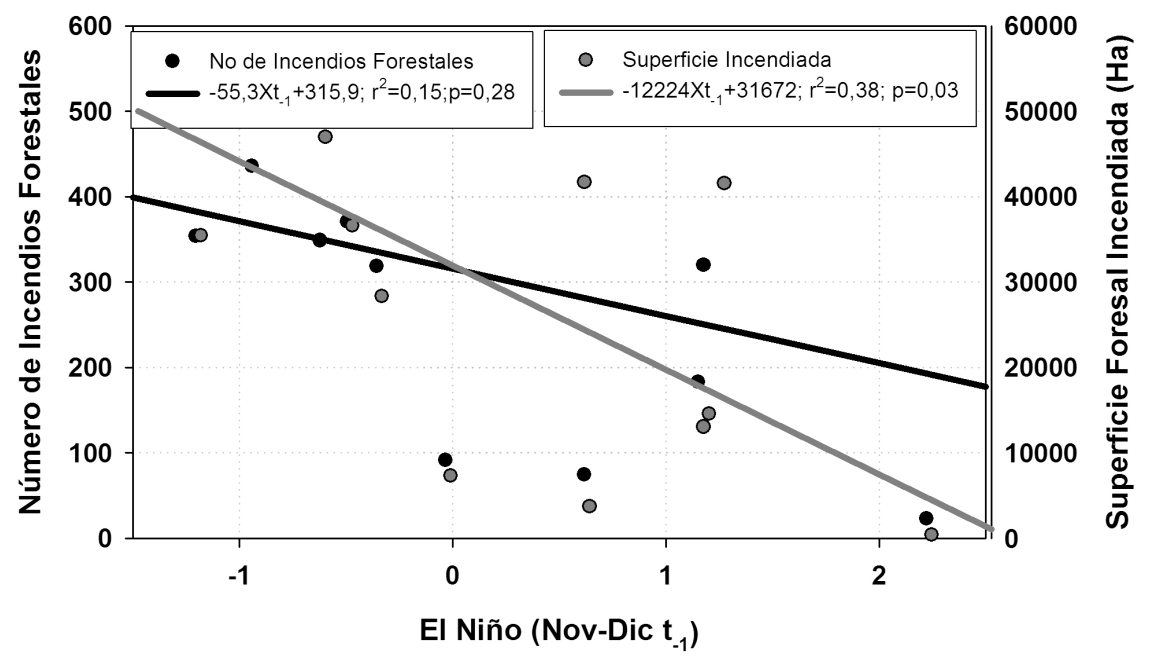

Figura 4. La importancia del fenómeno climático El Niño, sobre el número y superficie afectada por incendios forestales en Durango.

número y la superficie forestal incendiada disminuyen notoriamente hasta en un $50 \%$ del promedio. Estas condiciones resultan en teleconexiones de El Niño. En contraste, cuando se presenta un efecto La Niña, fuerte, la superficie forestal y el número de incendios forestales aumenta a las 50000 ha y los 400 incendios forestales anuales.

\section{DISCUSIÓN}

Los resultados de este estudio demuestran que el cálculo de la humedad del suelo a través de un balance hídrico es un indicador climático más efectivo que la misma precipitación, la evaporación y la evapotranspiración, que se utilizan generalmente como parámetros de entrada en 
la mayoría de los modelos de riesgo de incendios forestales. El uso de este parámetro incrementa la precisión en la proyección del número de incendios y la superficie forestal incendiada en el estado de Durango. El balance de masas, por ser un modelo físico puede extenderse a otros bosques con el simple cambio en los parámetros del suelo, de la vegetación, los factores climáticos y los factores de ponderación.

La figura 2 muestra que a medida que aumenta el número de días donde el suelo se encuentra más seco, $\theta<0,16 \mathrm{~cm}$ $\mathrm{cm}^{-1}$, el número de incendios aumenta rápidamente hasta alcanzar una constante o asíntota. En contraste, a medida que el número de días donde la humedad promedio del suelo aumenta, $\theta<0,16 \mathrm{~cm}$ $\mathrm{cm}^{-1}$, se requiere de un mayor número de días con estas características para que se presenten incendios forestales importantes en número y superficie. Sin embargo, la superficie forestal incendiada presenta una tendencia lineal para casi todos los rangos de humedad descritos.

Las ecuaciones de las figuras 2 y 3 predicen el número de incendios y la superficie forestal incendiada, casi convergen en aproximadamente 400 incendios por año y por las 40000 ha incendiadas anualmente. La consistencia en la convergencia en las ecuaciones explica que existen otros factores más allá del rango de la humedad del suelo que controlan el número y la superficie forestal afectada por incendios de alta envergadura. Es posible que una vez que el $\theta$ entre por debajo de la capacidad de campo o 33,3 kPa (1/3 de bar) de succión $\left(\theta=0,45 \mathrm{~cm} \mathrm{~cm}^{-1}\right)$ el suelo y el mantillo orgánico o los combustibles que se encuentran sobre el suelo se desequen a tasas diferenciales. Si esto es así, entonces las ecuaciones muestran solo la relación entre el contenido del agua del suelo-planta y la superficie y número de incendios forestales y no de los combustibles que se encuentran sobre el suelo. Por esta razón, parte de la variación inexplicable de los modelos de regresión puede encontrarse en el tipo de incendio forestal; es decir, una clasificación más estricta de incendios de copa y de mantillo del suelo podría eventualmente mejorar la bondad de ajuste de estas relaciones estadísticas. Es posible además que la humedad del suelo sea una variable que pronostica el número de incendios y la superficie forestal incendiada potencialmente. Entonces, en rodales con presencia de combustibles forestales sobre el suelo, los incendios de mantillo serían más frecuentes y posiblemente actuarían como quemas controladas en suelos relativamente húmedos. En rodales con contenido alto de combustibles forestales con suelos extremadamente secos, los incendios de copa, y por supuesto de suelo, serían los de mayor ocurrencia.

Las ecuaciones de regresión que predicen el número de incendios forestales son no lineales, en esencia, en contraste con aquellas que predicen la superficie forestal siniestrada. Es por ello importante clasificar las funciones que predicen cualquiera de estas dos variables importantes en la toma de decisiones sobre la prevención, el control y el manejo del fuego. La no linealidad de las ecuaciones es un hallazgo importante en este tipo de relaciones, ya que es un contraste a la suposición básica de linealidad de la mayoría de los modelos que predicen el riesgo de incendios forestales, utilizando valores climáticos en el submodelo (Aguado et al., 2003; Álvarez et al., 2006; Sebastián et al., 1999, 2000, 2007).

El balance hídrico puede utilizarse como un submodelo preliminar para la prevención de incendios forestales en la Sierra Madre Occidental. Este incluye todas las variables de entrada, salida y 
cambios en el almacenamiento del agua de los suelos. Es un adelanto en contraste con los modelos utilizados en la estimación del riesgo de incendios forestales, los cuales utilizan generalmente solo variables como la precipitación y la humedad relativa del aire (Sebastián et al., 2000; http://fire.cfs.nrcan.gc.ca; Hernández-Leal et al., 2005). Aunque existen algunos otros modelos que incorporan otros componentes del balance hídrico, como es la evapotranspiración (Sebastián et al., 1999), estos adolecen muy probablemente de falta de precisión en la estimación final del riesgo de incendios forestales.

El efecto del fenómeno climático El Niño se ha explicado por su influencia en la precipitación y la temperatura de varias regiones de la Tierra. Cuando éste se presenta en los últimos meses del año anterior y en los primeros meses del presente, generalmente durante el invierno y parte de la primavera, el norte de México es más frío y más lluvioso de lo normal (Cavazos y Hastenrath, 1990; Méndez-González et al., 2008). Swetnam y Betancourt (1989) en California y Arizona y Drury y Veblen (2007) en Durango, México, observaron que los incendios de mayor variación espacial estuvieron sincronizados con eventos climáticos extremos y estos se presentaron desfasadamente con el fenómeno climático El Niño. De tal manera que durante la fase cálida del Océano Pacífico oriental, el balance hídrico de los suelos es más positivo y benévolo para la productividad forestal (González-Elizondo et al., 2005; Arreola-Ortiz y NávarCháidez, 2010) y consecuentemente en la reducción del número y superficie que afectan los incendios forestales.

El Niño, en su fase alta, trae consigo altas precipitaciones durante el otoño del año anterior y el invierno y la primavera del presente año en muchas partes del sudoeste de Estados Unidos (Andrade y Sellers, 1988) y norte de México (Cavazos y Hastenrath, 1990; MéndezGonzález et al., 2008) que resulta de una fase intensa y profunda del Bajo Aleutiano (un desplazamiento hacia el sur de la ruta de tormentas del Pacífico Norte), en combinación con una fuerte corriente del chorro subtropical que trae humedad directamente de la zona de convergencia intertropical en el Pacífico centrooriental (Andrade y Sellers, 1988). Como ejemplo, estas condiciones prevalecieron en Durango a finales de 1996 y principios de 1997 durante la fase más intensa de $\mathrm{El}$ Niño del siglo pasado, cuando se registraron inclusive aguanieves en la Sierra Madre Occidental, a principios de mayo de 1997, y se han presentado en el 2010. Después de esta etapa el clima varía a seco y cálido con ondas de frío extremo, de tal manera que, por ejemplo, para fines de 1997 se presentaron las heladas más intensas del siglo pasado, con temperaturas por debajo de los $-25^{\circ} \mathrm{C}$, lo que ocasionó la mortalidad de numerosas especies exóticas y algunas nativas dentro de los bosques templados, aumentando la cantidad de combustibles sobre el suelo. Vientos fuertes y secos provenientes del Océano Pacífico, una primavera seca, desfasada un año de El Niño, combinado con cantidades importantes de combustibles propiciaron los incendios forestales más intensos de los últimos 50 años, en número y superficie incendiada en Durango, México. Una combinación del balance hídrico para suelos forestales con las proyecciones del fenómeno climático El Niño, podría eventualmente mejorar las estimaciones del índice de riesgo de incendios forestales en muchos bosques de México. Aquí se presenta un avance dentro del conocimiento de la presencia de incendios de alta envergadura y en combinación con técnicas estadísticas convencionales, como los cálculos de su probabilidad de ocurrencia (Alvarado et al., 1998) podrían eventual- 
mente mejorar sus predicciones en tiempo y espacio. Es por esta razón que se recomienda hacer uso de estas tecnologías con la finalidad de desarrollar estrategias importantes en la prevención de incendios forestales.

\section{CONCLUSIONES}

Este reporte de investigación enfatiza la necesidad de incorporar variables climáticas y edafológicas provenientes de balances hídricos en la proyección del número y superficie forestal incendiada en Durango. Estas variables proveen mayor información en la predicción del número y superficie forestal incendiada que las variables climáticas simples convencionalmente utilizadas en los modelos de predicción del riesgo de incendios forestales. Estas variables se expresan adecuadamente en fenómenos climáticos como El Niño-Oscilación Sur, y una combinación de la proyección de esta anomalía con las variables de los modelos hidrológicos, como es el balance del agua de suelos forestales, proveerían técnicas más adecuadas para la predicción de estos siniestros en los bosques templados del planeta.

\section{REFERENCIAS}

Aguado, I., E. Chuvieco, P. Martin y F.J. Salas. 2003. Assessment of forest fire danger conditions in southern Spain from NOAA images and meteorological indices. International Journal Remote Sensing 24:1653-1668.

Alvarado, E., D.V. Sandberg y S.G. Pickford. 1998. Modelling large forest fires as extreme events. Northwest Science 72:66-75.

Andrade, A.R. y W.D. Sellers. 1988. El Niño and its effect on precipitation in Arizona and Western New Mexico. Journal of Climatology 8:403-410.

Álvarez, E., C. de la Fuente y M.A. García. 2006. Índice de riesgo de incendios forestales para Aragón. In: 'V Simposio Nacional de Predicción'. INM. http://www.inm.es/web/sup/ciencia/div ulga/vsimposio/trabajos/pdf/C3ZAR_Incendios.pdf. 15/11/2007.

Arreola-Ortiz, M. y J.J. Návar-Cháidez. 2010. Análisis de sequías y productividad con cronologías de Pseudotsuga menziesii Rob \& Fern y su asociación con El Niño en el nordeste de México. Investigaciones Geográficas 71:7-20.

Bugalho, L. y L. Pessanha. 2007. The forest fire risk index (ICRIF) operational process and validation. In: "4a Conferencia Internacional sobre Incendios Forestales'. Organismo Autónomo de Parques Nacionales. Ministerio del Medio Ambiente. Sevilla, España, p. 8.

Cavazos, T. y S. Hastenrath. 1990. Convection and rainfall over Mexico and their modulation by the Southern Oscillation. International Journal of Climatology 10:377-386.

Conafor (Comisión Nacional Forestal). 2008. Estadísticas de los incendios forestales en México. México, D.F.

Drury, S.A. y T.T. Veblen. 2007. Spatial and temporal variability in fire ocurrent within the Las Bayas Forestry Reserve, Durango, Mexico. Plant Ecology 197:299-316.

Fiorucci, P., F. Gaetani y R. Minciardi. 2004. Sistema integrado para evaluar el riesgo estático y dinámico a nivel nacional de incendios forestales. In: '2nd Symposium on FIRE, Economics, Planning and Policy: A Global View'. Córdoba, España, pp. 1-20. 
Fulé, P.Z. y W.W. Covington. 1997. Fire regimes and forest structure in the Sierra Madre Occidental, Durango, Mexico. Acta Botánica Mexicana 41:43-79.

García, E. 1987. Modificaciones al sistema de clasificación climática de Köppen (para adaptarlo a las condiciones de la República Mexicana). $4^{a}$ ed. Instituto de Geografía, UNAM. México, 217 p.

Gash, J.H.C., C.R. Lloyd y G. Lachaud. 1995. Estimating sparse forest rainfall interception with an analytical model. Journal of Hydrology 170:79-86.

González-Elizondo, M., E. Jurado, J. Návar, M.S. González-Elizondo, J. Villanueva, O. Aguirre y J. Jiménez. 2005. Treerings and climate relationships for Douglas-fir chronologies from the Sierra Madre Occidental, México: a 1681-2001 rain reconstruction. Forest Ecology and Management 213:39-53.

Hillel, D. 1980. Fundamentals of Soil Physics. Academic Press. Londres, Reino Unido, 320 p.

Hernández-Leal, P.A., M. Arbelo y A. González-Calvo. 2005. FIRE risk assessment using satellite data. Advances in Space research 37:741746.

http://fire.cfs.nrcan.gc.ca

INEGI (Instituto Nacional de Geografía Estadística e Informática). 2010. Censo de Población y Vivienda. Durango, Dgo., México. $540 \mathrm{p}$.

Johnson, E.A. y K. Miyanishi. 2001. Forest fires. Behaviour and ecological effects. San Diego, Academic Press. EUA, 412 p.
Lawson, B.D., G.N. Dalrymple y B.C. Hawkes. 1997. Predicting forest floor moisture contents from Duff Moisture Code values. Canadian Forest Service, Pacific Forestry Centre, Victoria, BC. Technology Transfer Note 6.

Méndez-González, J., J. Návar y O.V. González. 2008. Análisis de tendencias de la precipitación (1920-2004) en México. Investigaciones Geográficas 65:38-55.

Návar, J. y R. Bryan. 1994. Fitting the analytical model of rainfall interception of Gash to semi-arid shrubs in northeastern Mexico. Agricultural and Forest Meteorology 68:133-143.

Návar, J., E. Carlyle-Moses y A.M. Martínez. 1999. Interception loss from the Tamaulipan matorral thornscrub of northeastern Mexico: an application of the gash analytical interception loss model. Journal of Arid Environments 40:1-10.

Návar, J., F. Charles y E. Jurado. 1999a. Spatial variations of interception loss components by Tamaulipan thornscrub in northeastern Mexico. Forest Ecology and Management 124:231-239.

Návar, J. 2008. Atlas Hidrológico del Estado de Durango. Inédito, 180 p.

Návar, J. 2011. The performance of Gash's revised interception loss model in Mexico's northeastern temperate forests. Aceptado con revisiones en Hydrological Processes.

Návar, J. 2011a. Stemflow variation in Mexico's northeastern forest communities: its contribution to soil moisture content and aquifer recharge. Journal of Hydrology 408: 35-42. 
Sebastián L.A., R.E. Burgan, A. Calle y A. Palacios-Orueta. 2007. Calibration of the fire potential index in different seasons and bioclimatic regions of southern Europe. In: '4a Conferencia Internacional sobre Incendios Forestales'. Organismo Autónomo de Parques Nacionales. Ministerio del Medio Ambiente. Sevilla, España.

Sebastián L.A., J. San Miguel-Ayanz y G. Libertá. 2000. An integrated forest fire risk index for Europe. In: "20th EARSeL Symp: Remote Sensing in the 21st Century: A decade of trans European remote sensing cooperation'. Dresden, Alemania, pp. 137-142.

Sebastián L.A., G. Libertá y J. San MiguelAyanz. 1999. An integrated forest fire risk index for European regions. Fire Potential Index. Joint Research Centre. www.etsea2.ud1.es.

Swetnam, T.W. y J.L. Betancourt. 1989. El Niño-Southern Oscillation (ENSO) phenomena and forest fires in the Southwestern United States. In: J.L. Betancourt y A.M. MacKay (eds.). 1990. Proceedings of the Sixth Annual Pacific Climate (PACLIM) Workshop. March 5-8, 1989. California Department of Water Resources, Interagency Ecological Studies Program. Technical Report 23.

Viessman, W.J., G.L. Lewis y J.W. Knapp. 2007. Introduction to Hydrology. 4a. ed. Harper \& Row. Nueva York, EUA, $780 \mathrm{p}$. 